\title{
In vitro survival of breast cancer cell lines following chemotherapy or radiotherapy in comparison with gold-mediated phototherapy
}

\author{
Moussa $\mathrm{S}^{1}$, Thanaa Shalaby1, Zaher $\mathrm{E}^{2}$, Elnaggar $\mathrm{M}^{3}$ and Sameh Nakhla ${ }^{1}$ \\ ${ }^{1}$ Department of Radiation Sciences, Medical Research Institute, Alexandria University, Alexandria, Egypt \\ ${ }^{2}$ Department of Medical Biophysics, Medical Research Institute, Alexandria University, Alexandria, Egypt \\ ${ }^{3}$ Department of Cancer Management and Research, Medical Research Institute, Alexandria University, Alexandria, Egypt
}

\section{III}

Background: Breast cancer is a heterogeneous disease that mandate efficient therapeutics, lower drug toxicity, and overcoming drug resistance. Combination therapy was suggested as a future treatment to attain the required efficacy and tolerable side effects. Aim: The current study aimed to compare and evaluate the efficacy of single and combined treatments in the MCF-7 breast cancer cell line. Materials and Methods: The study included seven MCF-7 groups according to the treatment modality. Cell viability was evaluated by MTT assay at different doses and time course treatments. Results: When nanoparticles were used alone, the starting point of significant cell death was $100 \mu \mathrm{g}$, but when using a photothermal combination modality the loss of viability \% was bout 2fold higher than nanoparticles alone especially in low concentrations and there was a significant difference between the 2 groups. There was a significant difference in cell viability between FAC + AuNPs group when compared to FAC only or control group $(p \leq 0.05)$. There was a significant difference in cell viability between Taxol + AuNPs when compared to control $(p \leq 0.05)$. there is a significant effect of radiation doses on cell viability within all subgroups ( $p<0.001$ for $R 0$, and $p=0.001$ for $R 2$ subgroup). there is a significant effect of the radiation doses on the cell viability within each subgroup as indicated by significant $p$ values ( $p=0.027$ for RNO, and $p=0.001$ for RN2). Conclusion: Combined GNPs and FAC, paclitaxel, and Radiotherapy treatment modalities could improve breast cancer outcomes and prognosis.

Keywords: Breast cancer; Chemotherapy; MCF-7; Photothermal treatment; Radiosensitizers

Editor-in-Chief: Prof. M.L. Salem, PhD - Article DOI: 10.21608/JCBR.2021.60568.1150 